\title{
Notas sobre la vegetación desértica del Parque Eólico Jepírachi, Alta Guajira, Colombia
}

\author{
Gabriel Pinilla-Agudelo*, Silvio Zuluaga-Ramírez \\ Departamento de Biología, Universidad Nacional de Colombia, Bogotá, Colombia
}

\begin{abstract}
Resumen
A fin de describir la vegetación del Parque Eólico Jepírachi (municipio de Uribia, Alta Guajira), se realizó un muestreo entre el 24 de septiembre y el 1 de octubre de 2001. En el área domina una comunidad vegetal compuesta por las especies Castela erecta, Prosopis juliflora y Opuntia caracasana. En general, la estructura presenta coberturas bajas, abundancias moderadas y portes reducidos. La mayor parte del matorral se distribuye en las terrazas, superficies de denudación y dunas, muy expuestas a vientos fuertes, los cuales ocasionan una xeromorfia acentuada y procesos de 'anemomorfósis' en las plantas.
\end{abstract}

Palabras clave: desierto, xeromorfosis, viento, anemomorfósis, Jepírachi, Alta Guajira.

Notes on desert vegetation of the Jepírachi Wind Farm in the Alta Guajira, Colombia

\begin{abstract}
In order to describe the vegetation of the Jepirachi windfarm (Uribia, Alta Guajira), samples were taken from 24 September to 1 October 2001. The plant community in the area is mainly composed of Castela erecta, Prosopis juliflora and Opuntia caracasana. In general, the predominant structure is of sparse coverage, moderate abundance and reduced height. Most of the scrubs are distributed on the terraces, denudation surfaces and dunes, very exposed to strong winds. Winds induce xeromorphic characteristics in plants, also causing processes of anemomorphosys.
\end{abstract}

Key words: Desert, xerophytia, wind, anemomorphosys, Jepírachi, Alta Guajira.

\section{Introducción}

El bioma desértico tropical de La Guajira se ha venido estudiando desde inicios de los años 40, con los aportes de Dugand (1941), que continuaron 20 años después en los estudios de Hernández, et al. (1963) y Espinal \& Montenegro (1963) y, posteriormente, en los de Rieger (1976). Recientemente, Rangel (2012) recopiló los aspectos florísticos de la región Caribe, trabajo en el cual incluyó una descripción fitosociológica que amplía los resultados del estudio de Rieger (1976) en la península.

Uno de los factores que modelan las formaciones y el paisaje en la Alta Guajira son los vientos extremadamente fuertes. El primer efecto directo del viento es el arrastre de la humedad del aire, pero, además, el viento es muy efectivo en el transporte de arena de cierto tamaño, lo que ejerce una acción abrasiva sobre el paisaje (Okin, et al., 2006). Igualmente, la formación de dunas y su movimiento se deben a dicho factor. A pesar de la importancia del viento en los desiertos, no se ha hecho suficiente énfasis en las respuestas de la vegetación a esta fuerza física y solo se ha documentado la forma en que las especies leñosas se achaparran y la deformación de la arquitectura de las plantas en el sentido en que sopla el viento (efecto bandera) (Hernández, et al., 1995a).

El objetivo del presente artículo es dar a conocer algunas características fisonómicas, florísticas y estructurales de la vegetación del Parque Eólico Jepírachi, ubicado en una de las zonas con mayor fuerza del viento en el país (Pinilla, $\boldsymbol{e t}$ al., 2009), el cual es muy importante por ser el primero de su clase en Colombia.

\section{Generalidades del área de estudio}

El Parque Eólico Jepírachi está ubicado en la Alta Guajira, cerca de Puerto Bolívar, al oeste de Bahía Portete (Figura 1), en el municipio de Uribia, departamento de La Guajira. Las coordenadas del sitio de estudio son $72^{\circ}$ este y $12^{\circ}$ 15' norte (Peláez, et al., 2004) y su extensión es de 3.000

\footnotetext{
*Correspondencia:

Gabriel Pinilla-Agudelo, gapinillaa@unal.edu.co

Recibido: 12 de agosto de 2013

Aceptado: 7 de marzo de 2014
} 


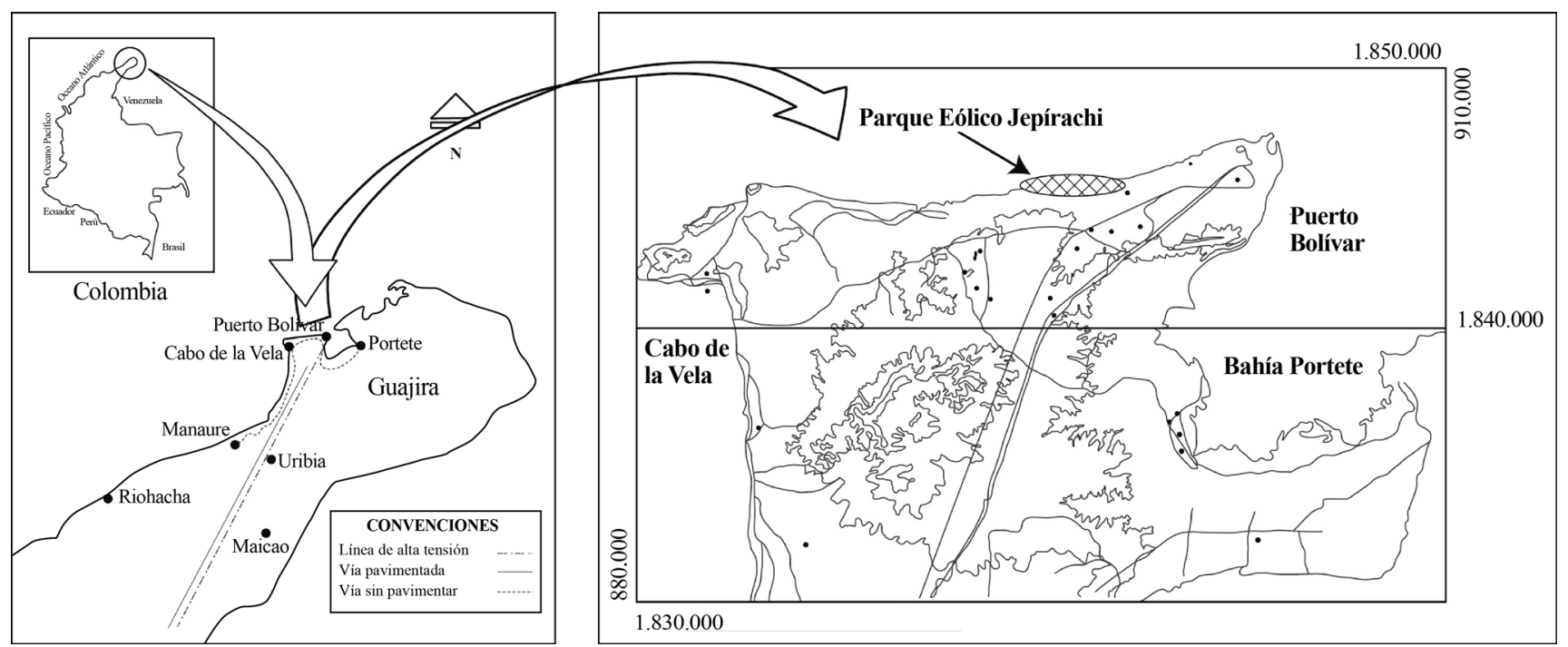

Figura 1. Ubicación del Parque Eólico Jepírachi en La Guajira, Colombia

hectáreas. Esta zona corresponde geológicamente a una mesa de areniscas calcáreas que se extiende desde el Cabo de la Vela hasta Puerto Estrella (IGAC, 1977). Localmente hay formaciones holocénicas de dunas de arena y depósitos aluviales a lo largo de los arroyos. También se dan, en sitios localizados, condiciones plano-depresionales con sedimentos altamente salinos. En el Cabo de la Vela y en Bahía Honda se reportan valores de 158 y $178 \mathrm{~mm}$ anuales de precipitación, respectivamente (IGAC, 1977); Rangel (2012) registra valores de 249,5 mm anuales para Puerto Bolívar y 221 para Puerto López, estaciones ubicadas en el municipio de Uribia. La temperatura media anual alcanza los $28{ }^{\circ} \mathrm{C}$ y la radiación solar es superior a $600 \mathrm{cal} \mathrm{cm}^{-2}$ día $^{-1}$ (IDEAM, 1998). El clima es, por lo tanto, desértico tropical, y sus efectos se observan con vigor en la zona de estudio (bioclima tropical xérico, Rivas-Martínez, 2008). En la Alta Guajira el viento sopla en dirección este-oeste y este-nordeste con velocidades que fluctúan entre 3 y $18 \mathrm{~m} / \mathrm{s}$ (Rodríguez, 2004). Entre marzo de 2004 y julio de 2005, Pinilla, et al. (2009) registraron en el parque Jepírachi un promedio de $9,2 \mathrm{~m} / \mathrm{s}$ entre 10 y $20 \mathrm{~m}$ de altura; según estos autores, el promedio mensual esperado a $60 \mathrm{~m}$ de altura es de $12 \mathrm{~m} / \mathrm{s}$; los promedios mensuales más bajos ocurren en septiembre y octubre, con velocidades de 6 y $6,5 \mathrm{~m} / \mathrm{s}$, respectivamente. Para el resto de la península de La Guajira la velocidad de los vientos oscila entre $5 \mathrm{~m} / \mathrm{s}$ y $11 \mathrm{~m} / \mathrm{s}$, y para Riohacha, el promedio anual es de $4 \mathrm{~m} / \mathrm{s}$ (UPME \& IDEAM, 2006). La fisiografía origina suelos de características típicas de aridisoles (Haplocambids, Haplocalcids y, en menor proporción, Haplosalids) y de entisoles desarrollados en los materiales arenosos de las dunas (Torripsamments) (EPM, 2002).
En lo relacionado con las condiciones socioeconómicas, la región se encuentra en el territorio del resguardo indígena de la comunidad Wayúu. Al parecer, la ocupación y el uso de esta región comenzaron hace 2.500 años (EPM, 2002).

\section{Las formaciones vegetales del área}

El área de estudio se encuentra dentro de la zona de vida del matorral desértico tropical, según la clasificación de Holdridge (1982), del Arid temperate life zone según Chapman (1917), y del bioclima tropical xérico, según Rivas-Martínez (2008). La formación dominante corresponde al matorral desértico (Hernández, et al., 1995b), que se divide, a su vez, en diferentes grupos de acuerdo a las características de las especies presentes en el lugar. En el área del parque eólico se encuentran el matorral desértico micrófilo con predominio del trupillo, Prosopis juliflora (Sw.) DC., el matorral desértico nanófilo, dominado por la matapuerca, Castela erecta Turp., el matorral desértico crasicaule, caracterizado por cactáceas como el cardón, Stenocereus griseus (Haw.) Buxb.,y la tuna, Opuntia caracasana Salm - Dyck, y el matorral desértico psammófilo, compuesto por plantas psamófitas y halófitas como la verdolaga de playa, Sesuvium portulacastrum (L.) L., y el platanito, Batis marítima L. Los dos primeros matorrales imperan en casi toda la zona del parque, pero el matorral nanófilo se intercala con los demás según las condiciones de exposición al viento y las características edáficas de cada sector (Hernández, et al., 1995b). El matorral psammófilo se presenta en algunas pequeñas áreas costeras, frente a la desembocadura de los arroyos, donde se forman dunas de condiciones húmedas. Hacia las terrazas se presentan dunas muy secas donde se encuentra Sporobolus virginicus (L.) Kunth y, ocasionalmente, S. griseus, Jatropha gossypiifolia 
L. y Calotropis procera (Aiton) Dryand., especies que ayudan a estabilizar y arraigar las dunas. Sobre $C$. procera, no obstante, hay que señalar que es una especie introducida que podría estar afectando el desarrollo de la sucesión natural de la zona. En los arroyos y en inmediaciones de los jagüeyes se presenta el matorral desértico micrófilo dominado por $P$. juliflora, pero con un mayor desarrollo de su altura y cobertura debido a las condiciones favorables de un mejor nivel freático y por estar al abrigo del viento en las áreas depresionales y en los jarillones.

El matorral desértico nanófilo es una formación extensa, rala y dispersa que cubre un paisaje amplio de condiciones fisiográficas y edáficas barridas por fuertes vientos, los cuales incrementan las características de sequedad y provocan una erosión eólica extrema sobre superficies planas elevadas y levemente basculadas hacia el mar, que corresponden a áreas de denudación y terrazas marinas (EPM, 2002). Se encuentran, también, llanuras costeras en el sector comprendido entre la ranchería Kasiwolín y el litoral, así como campos de dunas formados por acumulación de arena fina transportada por el viento, generalmente adyacentes a la franja costera.

\section{Materiales y métodos}

\section{Muestreo de la vegetación}

Se partió de una 'fotointerpretación' previa, en la cual se identificaron las unidades fisiográficas predominantes (superficies de denudación planas y disectadas, playas, dunas, terrazas, corredores aluviales) y en campo se ubicaron los sitios de muestreo. El muestreo se realizó entre el 24 de septiembre y el 1 de octubre de 2001. Se empleó el método del transecto en línea (Matteucci \& Colma, 1982), consistente en el censo detallado de la vegetación a lo largo de líneas de diferente longitud, la cual dependió de la extensión ocupada por la vegetación. De esta manera, en los matorrales de los planos de denudación el ancho fluctuó entre uno y cinco metros y el largo entre 40 y $120 \mathrm{~m}$, mientras que en los matorrales de los arroyos estas dimensiones oscilaron entre $1,5 \mathrm{~m}$ de ancho y 30 y $100 \mathrm{~m}$ de largo. En cada transecto se registró el número de individuos de las especies presentes. Se hicieron observaciones generales en torno a la fisonomía y se midieron la cobertura y la altura de las plantas en las intersecciones sobre la línea en cada sector del parque. La identificación taxonómica se realizó en el Herbario Nacional Colombiano del Instituto de Ciencias Naturales de la Universidad Nacional (sede Bogotá). El material se encuentra hoy en el depósito de dicho herbario.

\section{Análisis}

Para obtener las coberturas relativas de las especies, sus valores correspondientes en las intersecciones se tradujeron a porcentajes de acuerdo al área de cada transecto. Posteriormente, se hizo una segunda relativización teniendo en cuenta todos los transectos. El análisis de correspondencias linearizado (detrended correspondance analysis, DCA) se realizó con dichos valores de cobertura doblemente relativizada mediante el empleo del programa PC-ORD (McCune \& Mefford, 1995).

\section{Resultados y discusión}

\section{Fisonomía}

La comunidad estudiada en el área es de porte reducido (Figura 2) debido a las condiciones desfavorables del suelo, la escasez de agua y los vientos fuertes (EPM, 2002). La fisonomía se ve influenciada notoriamente por

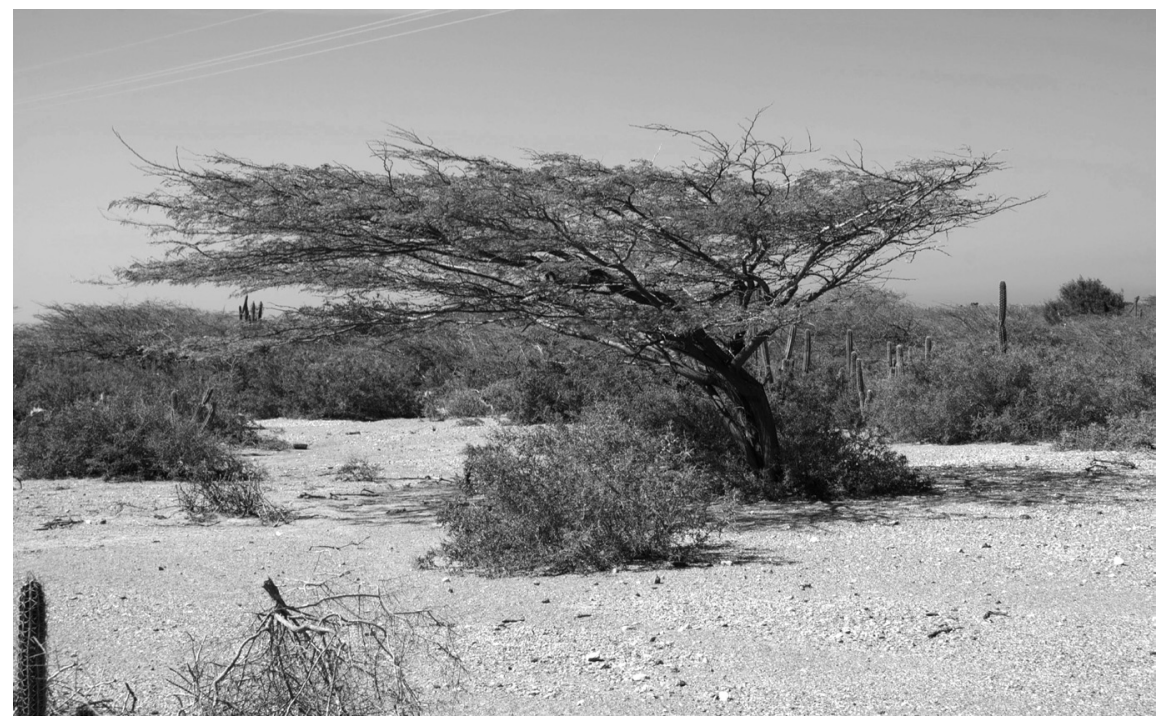

Figura 2. Comunidad de Castela erecta del parque eólico Jepírachi, en la cual se aprecia el "abanderamiento" de un ejemplar de Prosopis juliflora. 
la exposición al viento, el cual ejerce una fuerte presión de adaptación por anemomorfósis (Váczy, 1980) y provoca en las plantas respuestas como el 'abanderamiento'. El "efecto bandera", estudiado desde hace tiempo en sitios como las islas Británicas (Oliver, 1960) y Hawai (Noguchi, 1979), constituye un alargamiento del follaje de la planta en el sentido en que predomina la dirección del viento y es mayor en aquellas áreas donde la fuerza y la velocidad del viento son elevadas, es decir, en los sitios más expuestos a la acción eólica. Este fenómeno es común en árboles y arbustos como P. juliflora (Figura 2) y Parkinsonia praecox (Ruiz \& Pav.) Hawkins, pero se presenta incluso en plantas bajas como $C$. erecta. El efecto bandera es uno de los fenómenos que ocurren en todos los matorrales desérticos del área, en especial en sitios de alta exposición al viento, con velocidades promedio de $9,2 \mathrm{~m} / \mathrm{s}$ (Pinilla, et al., 2009).

La fuerza del viento ejerce otro efecto importante sobre la fisonomía, el cual se manifiesta regularmente en $O$. caracasana, cuyos cladodios, o segmentos del tallo articulados unos con otros, se disponen de tal manera que sus filos o cantos se sitúan en contra del viento, reduciendo así el área de contacto y evitando la posible caída de la planta cuando el viento es muy fuerte. Para este efecto se propone la denominación de "enfilamiento", el cual se puede observar en la figura 3, en la cual se observa cómo las plantas de $O$. caracasana tienden a estar en un solo plano.
Las adaptaciones generales a las condiciones desérticas en estas zonas de alta exposición y velocidad del viento se ven incrementadas en las manifestaciones fisonómicas por la carencia de agua, y están presentes también en otro tipo de formaciones edáficas con acentuado déficit hídrico a través del xeromorfismo (Larcher, 1975). Algunas de tales estrategias ante la aridez son la suculencia, la microfilia y la pérdida temporal o estacional del follaje (Hernández, et al., 1995a; Allaby, 2001). Existen otros tipos de adaptaciones morfológicas que no necesariamente responden a las condiciones usuales de aridez del área, entre las cuales se pueden mencionar las observadas en las plantas del Parque Eólico Jepírachi que mencionamos a continuación.

Tortuosidad: formación de intrincados y complejos laberintos de ramas y ramitas que se entrecruzan en todas las direcciones. Esta puede ser una respuesta a la defoliación periódica, pero también genera estructuras más resistentes a la fuerza del viento. Es muy común en P. juliflora, C. erecta, Bursera sp. Jacq. ex L. y P. praecox, especialmente en los matorrales de las terrazas y de los arroyos.

'Aparasolamiento': formación de una copa plana o "parasol", a manera de pagoda, en la que el follaje se ubica en el plano superior y presenta menor resistencia al viento. Esta característica es propia de $P$. juliflora, la cual se encuentra en los matorrales de los jagüeyes y de los arroyos, donde hay cierta protección contra los efectos eólicos.

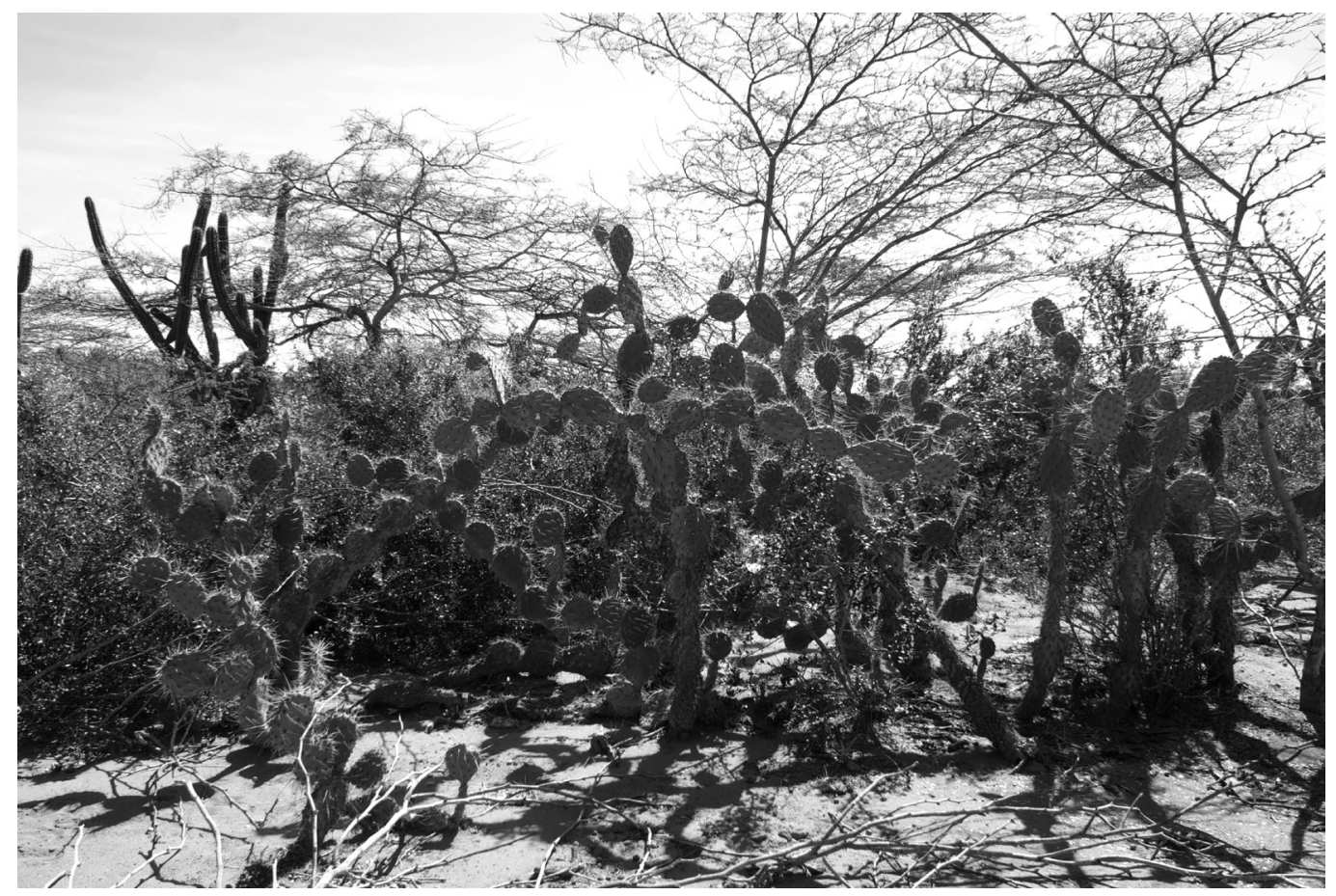

Figura 3. Efecto de "enfilamiento" en Opuntia caracasana. Nótese la ubicación de la mayoría de los cladodios de las tunas en un solo plano, en el cual los cantos se ubican contra el viento. 
'Macollamiento': formación de estructuras semejantes a macollas, alargadas en la dirección del viento y de baja altura. En las raíces de estas estructuras se acumula arena, formando pequeños montículos que favorecen su consolidación, tal como ocurre en los matorrales de las dunas. Estas estructuras son frecuentes en S.virginicus.

'Achaparramiento': este fenómeno ocurre en la mayoría de las especies afectadas por el viento, aunque hay otros factores asociados como la sequía, las restricciones edáficas y nutricionales, la neblina y la escasa radiación solar (Lamberts, et al., 2008). Dicho enanismo en las plantas es más común en los matorrales desérticos de las superficies de denudación, de las terrazas, de las dunas altas y de las playas. En $C$. erecta se presenta con mucha frecuencia, principalmente cuando se encuentra en condiciones de exposición al viento como los matorrales desérticos de superficies de denudación y de terrazas y dunas bajas.

Crecimiento postrado: las plantas se desarrollan y crecen a ras del suelo, de manera tal que parecen formar un tapete, con lo que reducen la resistencia a la acción del viento. Esta estrategia se observa en J.gossypiifolia, P. juliflora y C. erecta, en especial cuando hacen parte de los matorrales desérticos de las superficies de denudación y de las dunas bajas. S. portulacastrum, del matorral de las dunas, también desarrolla esta estrategia.

Tabla 1. Composición de los diferentes transectos de vegetación en el área del Parque Eólico Jepírachi - Guajira. Los valores de las especies corresponden a la cobertura relativa y al número de individuos a la derecha. Las especies se ordenaron según la frecuencia absoluta. Colector-número GPCV: Gabriel Pinilla - Cabo de la Vela

\begin{tabular}{|c|c|c|c|c|c|c|c|c|}
\hline $\begin{array}{l}\text { No. del } \\
\text { transecto }\end{array}$ & 1 & 2 & 3 & 4 & 5 & 6 & 7 & 8 \\
\hline Localidad & $\begin{array}{l}\text { Arroyo } \\
\text { Apure } 1\end{array}$ & $\begin{array}{l}\text { Arroyo } \\
\text { Apure } 2\end{array}$ & $\begin{array}{c}\text { Arroyo } \\
\text { Taruásaru }\end{array}$ & Mushalerrain & $\begin{array}{l}\text { Jagüey en } \\
\text { Arütkajüi }\end{array}$ & Arütkajüi & $\begin{array}{l}\text { Dunas altas } \\
\text { en Arütkajüi }\end{array}$ & $\begin{array}{l}\text { Dunas bajas } \\
\text { en Arütkajüi }\end{array}$ \\
\hline $\begin{array}{l}\text { Longitud del } \\
\text { transecto (m) }\end{array}$ & 30 & 100 & 30 & 90 & 120 & 100 & 120 & 100 \\
\hline $\begin{array}{l}\text { Área del } \\
\text { transecto }\left(\mathrm{m}^{2}\right)\end{array}$ & 45 & 1000 & 300 & 90 & 575 & 200 & 805 & 100 \\
\hline $\begin{array}{l}\text { Unidad } \\
\text { fisiográfica }\end{array}$ & Arroyo 1 & Arroyo 2 & Arroyo 3 & Superficie disectada 1 & Jagüey & $\begin{array}{l}\text { Superficie } \\
\text { plana } 1\end{array}$ & Dunas altas & Dunas bajas \\
\hline Castela erecta & $2,33-4$ & & $0,21-1$ & $8,7-28$ & $3,93-12$ & $9,5-24$ & $0,26-1$ & $52,99-15$ \\
\hline Prosopis juliflora & $41,37-21$ & $68,05-19$ & $84,66-15$ & $3,92-8$ & $54,16-13$ & $18,58-7$ & $24,69-79$ & \\
\hline $\begin{array}{l}\text { Opuntia } \\
\text { caracasana }\end{array}$ & $12,17-16$ & & $0,62-13$ & & $0,02-5$ & $0,018-4$ & $0,006-1$ & \\
\hline $\begin{array}{l}\text { Stenocereus } \\
\text { griseus }\end{array}$ & & & & $0,05-4$ & & $0,22-1$ & & $0,06-1$ \\
\hline $\begin{array}{l}\text { Jatropha } \\
\text { gossypiifolia }\end{array}$ & $9,42-32$ & & & & & $0,14-1$ & & $13,98-113$ \\
\hline $\begin{array}{l}\text { Lycium } \\
\text { tweedianum }\end{array}$ & $1,48-4$ & & $0,001-1$ & $0,68-4$ & & & $11,42-39$ & \\
\hline Melocatus sp. & & & & $0,002-1$ & & & & \\
\hline $\begin{array}{l}\text { Parkinsonia } \\
\text { praecox }\end{array}$ & & & & & & $4,02-3$ & & \\
\hline \multicolumn{9}{|l|}{$\begin{array}{l}\text { Pereskia } \\
\text { guamacho }\end{array}$} \\
\hline Bursera sp. & & & $2,66-1$ & & & & & \\
\hline $\begin{array}{l}\text { Cnidosculus } \\
\text { urens }\end{array}$ & $1,37-4$ & & & & & & & \\
\hline $\begin{array}{l}\text { Caesalpinia } \\
\text { coriaria }\end{array}$ & & & & $0,83-2$ & & & & \\
\hline $\begin{array}{l}\text { Sesuvium } \\
\text { portulacastrum }\end{array}$ & & & & & & & $0,005-1$ & \\
\hline $\begin{array}{l}\text { Haematoxylum } \\
\text { brasiletto }\end{array}$ & & & & $0,03-1$ & & & & \\
\hline
\end{tabular}




\begin{tabular}{|c|c|c|c|c|c|c|c|c|}
\hline No. del transecto & 9 & 10 & 11 & 12 & 13 & & & \\
\hline Localidad & $\begin{array}{l}\text { Torre EPM en } \\
\text { Arütkajüi }\end{array}$ & Kasushi 1 & Kasushi 2 & Kasiwolin & $\begin{array}{l}\text { Terrazas en } \\
\text { Kasiwolin }\end{array}$ & No tot ind. & $\begin{array}{c}\text { Frecuencia } \\
\text { absoluta }\end{array}$ & $\begin{array}{l}\text { Colector- } \\
\text { número }\end{array}$ \\
\hline $\begin{array}{l}\text { Longitud del } \\
\text { transecto (m) }\end{array}$ & 60 & 40 & 80 & 60 & 120 & & & \\
\hline $\begin{array}{l}\text { Área del } \\
\text { transecto }\left(\mathrm{m}^{2}\right)\end{array}$ & 120 & 80 & 160 & 120 & 360 & & & \\
\hline $\begin{array}{l}\text { Unidad } \\
\text { fisiográfica }\end{array}$ & Superficie plana 2 & Superficie plana 3 & $\begin{array}{l}\text { Superficie } \\
\text { plana } 4\end{array}$ & $\begin{array}{l}\text { Superficie } \\
\text { disectada } 2\end{array}$ & Terraza & & & \\
\hline Castela erecta & $15,71-17$ & $3,34-6$ & $1,95-7$ & $7,54-18$ & $12,5-48$ & 181 & 12 & GPCV13 \\
\hline Prosopis juliflora & & $23,13-14$ & $6,36-5$ & $7,26-4$ & $0,77-2$ & 187 & 11 & GPCV8 \\
\hline $\begin{array}{l}\text { Opuntia } \\
\text { caracasana }\end{array}$ & $0,33-18$ & $5,1-57$ & $0,3-18$ & $2,29-19$ & & 151 & 9 & GPCV2 \\
\hline $\begin{array}{l}\text { Stenocereus } \\
\text { griseus }\end{array}$ & $0,18-11$ & $0,21-8$ & $0,3-2$ & $0,05-3$ & $0,001-2$ & 32 & 8 & GPCV7 \\
\hline $\begin{array}{l}\text { Jatropha } \\
\text { gossypiifolia }\end{array}$ & & & & & $0,008-1$ & 147 & 4 & GPCV5 \\
\hline $\begin{array}{l}\text { Lycium } \\
\text { tweedianum }\end{array}$ & & & & & & 48 & 4 & GPCV1 \\
\hline Melocatus sp. & $0,03-1$ & $0,125-5$ & & & $0,003-1$ & 8 & 4 & GPCV10 \\
\hline $\begin{array}{l}\text { Parkinsonia } \\
\text { praecox }\end{array}$ & & & $2,5-2$ & & & 5 & 2 & GPCV14 \\
\hline $\begin{array}{l}\text { Pereskia } \\
\text { guamacho }\end{array}$ & & & $1,25-1$ & & & 2 & 2 & GPCV4 \\
\hline Bursera sp. & & $3-1$ & $5-1$ & & & 2 & 2 & GPCV9 \\
\hline $\begin{array}{l}\text { Cnidosculus } \\
\text { urens }\end{array}$ & & & & & & 4 & 1 & GPCV3 \\
\hline $\begin{array}{l}\text { Caesalpinia } \\
\text { coriaria }\end{array}$ & & & & & & 2 & 1 & GPCV12 \\
\hline $\begin{array}{l}\text { Sesuvium } \\
\text { portulacastrum }\end{array}$ & & & & & & 1 & 1 & GPCV6 \\
\hline $\begin{array}{l}\text { Haematoxylum } \\
\text { brasiletto }\end{array}$ & & $3,34-6$ & & & & 1 & 1 & GPCV11 \\
\hline
\end{tabular}

\section{Composición florística}

La vegetación del parque eólico corresponde a la amplia dominancia de la comunidad de P. juliflora, C. erecta, $O$. caracasana y $J$. gossypiifolia, especies que ostentan el mayor número de individuos (Tabla 1). Entre las especies frecuentes se encuentran $S$. griseus y Lycium tweedianum Griseb., y especies poco frecuentes como Melocactus curvispinus Pfeiff. (en la región solo se encuentra esta especie del género Melocactus según el estudio de Fernández \& Xhonneux, 2002), P. praecox, Cnidosculus urens (L.) Arthur, Pereskia guamacho F.A.C.Weber, Caesalpinia coriaria (Jacq.) Willd, Bursera sp., S. portulacastrum y Haematoxylum brasiletto $\mathrm{H}$. Karst.

Es notoria la sociabilidad de algunas especies vegetales como O. caracasana, asociada a P. juliflora y a C. erecta en los matorrales de las superficies de denudación, al igual que
L. tweedianum a los arbustos de P. juliflora en los matorrales de las dunas altas. En los matorrales de las dunas bajas, $J$. gossypiifolia se asocia a las macollas de C. erecta.

C. erecta es un arbusto espinoso de hojas caducas y nanófilas que junto con $P$. juliflora y $O$. caracasana conforma un matorral que corresponde a la clase Opuntio caracasanae - Prosopietea juliflorae, al orden Parkinsonio praecocis - Casteletalia erectae y a la alianza Stenocereo grisei Castelion erectae (Rangel y Garay, en Rangel, 2012). Las asociaciones descritas por Rangel (2012) para esta alianza son Caesalpinio mollis-Burseretum tomentosae, Subpilocereo repandi-Casteletum erectae y Melochio crenatae-Casteletum erectae. Muy probablemente la comunidad del Parque Eólico Jepírachi corresponda a una nueva asociación de Lycium y Castela que deberá ser estudiada detalladamente en investigaciones posteriores. L. tweedianum representa 
la especie característica exclusiva de esta comunidad (Tabla 1). Existe otra posibilidad para la denominación de esta comunidad, ya que $J$. gossypiifolia no es una especie exclusiva de la asociación Melochio crenatae-Casteletum erectae, pues se presenta también en la comunidad del parque Jepírachi. De esta manera, la posible asociación de Lycium y Castela podría ser una variante de una asociación más amplia y extensa en la que $J$. gossypiifolia podría ser un taxón exclusivo.

\section{Distribución y estructura}

La distribución de la vegetación del parque eólico obedece a dos aspectos fisiográficos de la región. En el primero, los sectores más húmedos se encuentran disectados por pequeños valles que albergan arroyos y arroyuelos protegidos de la acción muy fuerte de los vientos. En el segundo, los sectores más altos están expuestos a la acción eólica en terrazas y superficies de denudación.

En la figura 4 se puede observar una distribución de las especies basada en la cobertura y la posición fisiográfica, desde las áreas más húmedas a las más secas y expuestas al viento. Así, la porción más inferior de la figura indica las posiciones más húmedas, como los arroyos y el jagüey, y las superficies planas, con influencia de aguas freáticas en Arütkajüi y Kasushi 2, donde $P$. juliflora exhibe su mayor cobertura. Otras especies que muestran una clara influencia freática en la superficie plana de Kasushi 2 son Cnidosculus

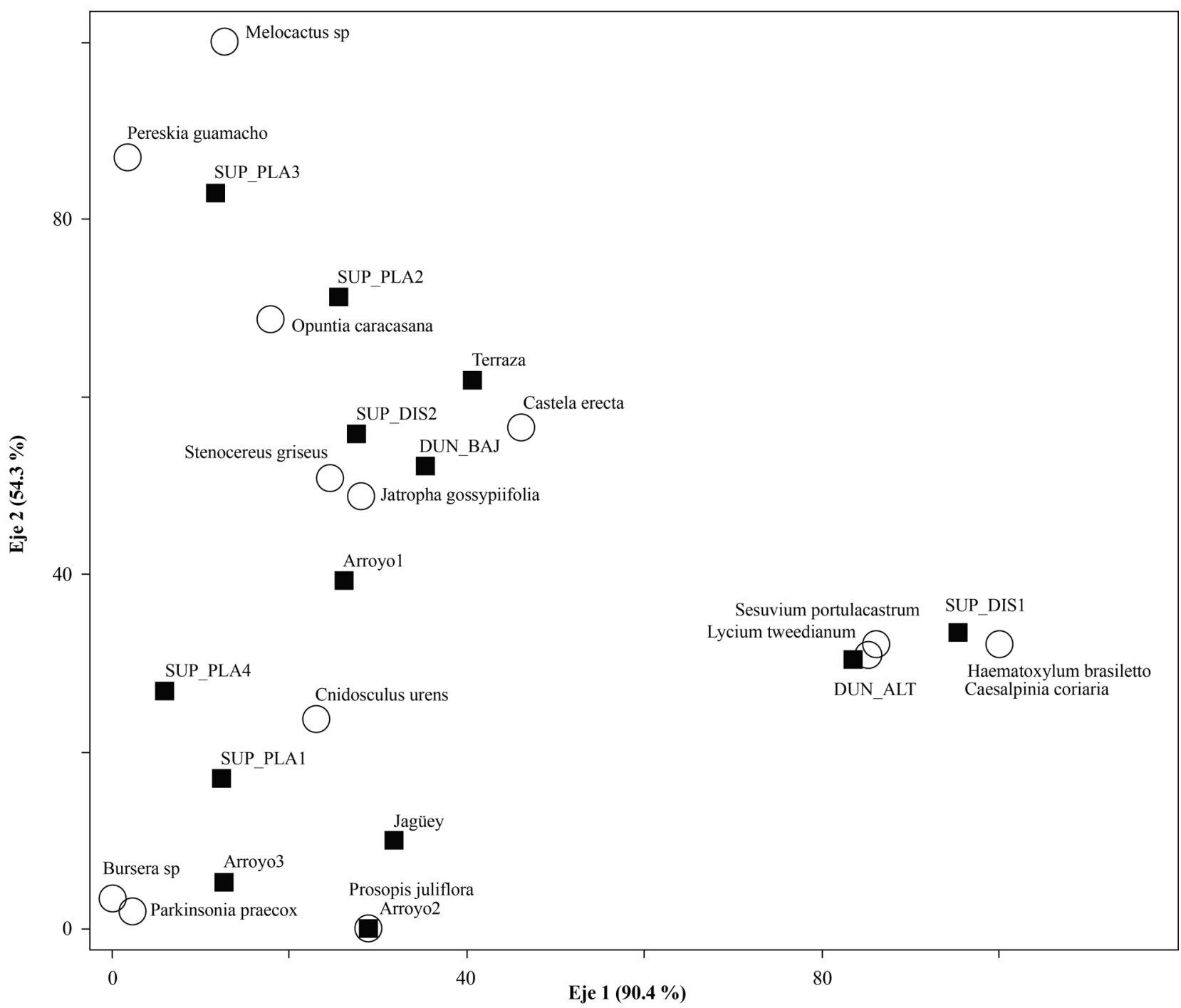

Figura 4. Ordenamiento DCA de la vegetación del Parque Eólico Jepírachi. Los círculos corresponden a las especies y los cuadrados a los sitios en las diferentes unidades fisiográficas. DUN ALT: dunas altas; DUN BAJ: dunas bajas; SUP-PLA1, 2, 3 y 4: superficies planas de denudación; SUP_DIS1 y 2: superficies disectadas. 
urens, P. praecox y Bursera sp. En la porción superior de la figura se aprecian la terraza, las superficies planas de la torre en Arütkajüi y de Kasushi 1, la superficie disectada de Kasiwolin y de las dunas bajas, donde, probablemente, la influencia de las aguas freáticas ha desaparecido $\mathrm{y}$ representan las posiciones más expuestas a la acción eólica. Entre las especies con mayor cobertura en estas áreas de extrema aridez se encuentran M. curvispinus, P. guamacho, O. caracasana, C. erecta, S. griseus y J. gossypiifolia. A la derecha de la figura se encuentran las dunas altas y la superficie disectada en Mushalerrain, donde las especies $S$. portulacastrum, H. brasiletto, C. coriaria y L. tweedianum representan los sitios más extremos donde se registran las menores coberturas.

En general, las especies $P$. juliflora y $C$. erecta se caracterizan por una cobertura dominante en el área del parque (Tabla 1). Dicha cobertura está afectada por el ramoneo del ganado ovino, caprino y caballar, que afecta ostensiblemente el desarrollo de la vegetación. Las especies mencionadas aportan, en su conjunto, el mayor número de individuos de la comunidad. Otras especies con alto número de individuos son O. caracasana y J. gossypiifolia.

La altura o porte promedio de las plantas en cada uno de los transectos del parque Jepírachi se aprecia en la Tabla 2. Es muy reducida y oscila entre $0,34 \mathrm{~m}$ en las superficies

Tabla 2. Altura promedio de la vegetación de los distintos transectos realizados en el Parque Eólico Jepírachi, Alta Guajira

\begin{tabular}{|c|c|c|}
\hline Transecto & Fisiografía & Altura promedio (m) \\
\hline Arroyo Apure 1 & Arroyo & 0,79 \\
\hline Arroyo Apure 2 & Arroyo & 4,14 \\
\hline Arroyo Taruásaru & Arroyo & 1,74 \\
\hline Mushalerrain & $\begin{array}{l}\text { Superficie de } \\
\text { denudación disectada }\end{array}$ & 0,34 \\
\hline Jagüey en Arütkajüi & Jagüey & 1,38 \\
\hline Arütkajüi & $\begin{array}{l}\text { Superficie de } \\
\text { denudación plana }\end{array}$ & 0,62 \\
\hline $\begin{array}{l}\text { Dunas altas en } \\
\text { Arütkajüi }\end{array}$ & Dunas altas & 0,90 \\
\hline $\begin{array}{l}\text { Dunas bajas en } \\
\text { Arütkajüi }\end{array}$ & Dunas bajas & 0,35 \\
\hline $\begin{array}{l}\text { Torre EPM en } \\
\text { Arütkajüi }\end{array}$ & $\begin{array}{l}\text { Superficie de } \\
\text { denudación plana }\end{array}$ & 0,39 \\
\hline Kasushi 1 & $\begin{array}{l}\text { Superficie de } \\
\text { denudación plana }\end{array}$ & 1,09 \\
\hline Kasushi 2 & $\begin{array}{l}\text { Superficie de } \\
\text { denudación plana }\end{array}$ & 1,48 \\
\hline Kasiwolin & $\begin{array}{l}\text { Superficie de } \\
\text { denudación disectada }\end{array}$ & 0,64 \\
\hline Terrazas en Kasiwolin & Terrazas & 0,38 \\
\hline
\end{tabular}

disectadas y 4,14 $\mathrm{m}$ en los arroyos. Los matorrales de los arroyos crecen un poco más y los árboles, protegidos del viento y con disponibilidad de agua freática, pueden superar los cuatro metros. Como puede verse en la Tabla 2, la altura varía de acuerdo a la posición fisiográfica del transecto; este es un factor importante a tener en cuenta en el impacto eólico sobre la vegetación, de tal forma que las superficies más expuestas, como dunas y terrazas, registran las alturas más reducidas. Por el contrario, las posiciones fisiográficas menos expuestas, como los arroyos y los jagüeyes, exhiben alturas ostensiblemente mayores. En las superficies de denudación planas de Kasushi, S. griseus, O. caracasana y $P$. guamacho alcanzan un porte ligeramente mayor. Es sorprendente que estas áreas, sujetas a una alta exposición al viento, presenten especies con alturas levemente mayores, en promedio, como $P$. guamacho, la cual alcanza más de $2 \mathrm{~m}$ de altura, probablemente porque con su sistema radical más desarrollado aprovecha mejor la disponibilidad de agua en el suelo, en comparación con las especies arbustivas.

\section{Consideraciones finales}

La acentuación de los factores que determinan los fenómenos de xeromorfosis (Font Quer, 1988) en La Guajira se pone claramente de manifiesto en la fisonomía de la vegetación del Parque Eólico Jepírachi. Los efectos de la xeromorfosis se evidencian fundamentalmente en los rasgos morfológicos de las plantas sometidas al estrés hídrico predominante y constante a lo largo del año. Sumado a este, otros factores como la excesiva velocidad del viento, pueden causar fenómenos de anemomorfósis (Váczy, 1980), pues la abrasión física limita el desarrollo de las plantas y provoca su crecimiento achaparrado típico en toda la zona. La acción del viento no solo produce cambios en la forma y la fisonomía de la vegetación, sino que el transporte de arena y otras partículas finas erosionadas del suelo puede provocar necrosis $\mathrm{y}$ rupturas, así como sepultar los individuos vegetales. A largo plazo, la acción del viento puede llevar al marchitamiento de las plantas más expuestas y a la eliminación de las ramas que sobresalen del dosel.

En las regiones costeras, los vientos arrastran niebla marina rica en sales (Larcher, 1975), lo que incrementa el estrés hídrico al que están sometidas las plantas. Todos estos factores, sumados a los tensores edáficos, han llevado al establecimiento de una comunidad vegetal en el Parque Eólico Jepírachi que puede considerarse como extremófila. Por supuesto, no solo el viento es el causante de este tipo de anemomorfósis. Hay otros factores, tales como el estrés hídrico y la limitación de nutrientes, que contribuyen a generar estos cambios drásticos en las formas de la vegetación. No obstante, la acción del viento parece ser la explicación principal de las adaptaciones morfológicas de las plantas del Parque Eólico Jepírachi. 
Desde el punto de vista florístico, en el parque eólico predominan las especies propias de la clase Opuntio caracasanea - Prosopietea juliflorae y de la alianza Stenocereo grisei - Castelion erectae descritas por Rangel (2012). Dadas las condiciones extremófilas descritas, sobresalen en el área especies de una sola comunidad, muy homogénea y siempre compuesta por taxones de la clase y la alianza mencionadas. Esta comunidad presenta una especie característica (L. tweedianum), la cual podría conferirle el estatus de asociación, por lo que se propone como una posible nueva asociación de L. tweedianum y C. erecta.

Las condiciones de disponibilidad de agua según la fisiografía de la zona determinan el porte de la vegetación y su cobertura. De esta manera, la vegetación de mayor porte y follaje más denso se confina a las áreas más protegidas o bajas de la fisiografía, y la de porte mínimo con una cobertura rala, a las áreas más expuestas y con poca disponibilidad de agua freática. Los portes y coberturas intermedios se hallan tanto en los sitios expuestos como en los protegidos, pero, de todas maneras, allí donde las plantas disponen de aguas freáticas.

\section{Agradecimientos}

Este trabajo se realizó en el marco del contrato No. 030211727 firmado entre las Empresas Públicas de Medellín y FAL Ltda. Ingenieros. Se agradece a estas instituciones la financiación del estudio y su invaluable colaboración durante su desarrollo.

\section{Conflicto de interés}

Los autores declaran no tener ningún conflicto de interés.

\section{Bibliografía}

Allaby M. 2001. Deserts. Facts on File Inc., Nueva York.

Chapman F. 1917. The distribution of bird life in Colombia: A contribution to a biological survey of South America. Bulletin of the American Museum of Natural History 36: $1-729$.

Dugand G. 1941. Estudios geobotánicos colombianos: descripción de una sinecia típica en la subxerofitia del Litoral Caribe. Revista de la Academia Colombiana de Ciencias Exactas, Físicas y Naturales, 4(14): 135-141.

Empresas Públicas de Medellín (EPM). 2002. Parque eólico piloto Jepírachi. Estudio de impacto ambiental. Informe Final. 2 volúmenes. Empresas Públicas de Medellín,Medellín.

Espinal L., Montenegro, E. 1963. Formaciones vegetales de Colombia: memoria explicativa sobre el mapa ecológico. Departamento Agrológico, Instituto Geográfico Agustín Codazzi, Bogotá.

Fernández, J.L., Xhonneux, G.2002. Novedades taxonómicas y sinopsis del género Melocactus Link. \& Otto. (Cactaceae) en Colombia. Revista de la Academia Colombiana de Ciencias Exactas, Físicas y Naturales, 26 (100): 353-365.

Font Quer, P. 1988. Diccionario de Botánica. Editorial Labor, Barcelona.

Hernández, J., Saravia, C., Jaramillo, R. 1963. Aspectos biogeográficos y biológicos. Parte II-B. En: Molano J. (ed). Conferencia Latinoamericana para el Estudio de las Zonas Áridas. Informe Nacional. Imprenta Nacional, Bogotá. pp. 49-63.

Hernández, J. 1991. Ensayo preliminar sobre los biomas terrestres de Colombia. En: SánchezH., J. Hernández, J.V. Rodríguez, Castaño, C. (eds.). Nuevos Parques Nacionales de Colombia. INDERENA, Bogotá. pp. 28-50.

Hernández, J., Rueda, V., Sánchez, H. 1995a. El bioma del desierto y las zonas áridas. En: Hernández J., Samper, D. (eds.). Desiertos. Zonas Áridas y Semiáridas de Colombia. Banco de Occidente, Bogotá. pp. 23-110.

Hernández, J., Rueda, V., Sánchez, H. 1995b. Zonas áridas y semiáridas de Colombia. En: Hernández J., Samper, D. (eds.). Desiertos. Zonas Áridas y Semiáridas de Colombia. Banco de Occidente, Bogotá. pp. 111-162.

Holdridge, L. 1982. Ecología basada en zonas de vida. IICA, San José de Costa Rica.

Instituto de Hidrología, Meteorología y Estudios Ambientales (IDEAM). 1998. El medio ambiente en Colombia. Instituto de Hidrología, Meteorología y Estudios Ambientales, Bogotá.

Instituto Geográfico Agustín Codazzi (IGAC). 1977. Zonas de Vida o Formaciones Vegetales de Colombia. Memoria explicativa sobre el Mapa Ecológico. Instituto Geográfico Agustín Codazzi, Subdirección Agrológica, Bogotá.

Lambers, H., Chapin, F.S. (III), Pons, T.L. 2008. Plant physiological ecology. Springer, The Hague.

Larcher, W. 1975. Physiological plant ecology. Springer-Verlag, New York.

McCune, B., Mefford, M.J. 1995. PC-ORD. Multivariate analysis of ecological data. Version 2.0. MjM Software, Gleneden Beach.

Matteucci, S., Colma, A. 1982. Metodologías para el estudio de la vegetación. Organización de Estados Americanos, Monografía No 22, Serie Biología, Washington.

Noguchi, Y. 1979. Deformation of trees in Hawaii and its relation to wind. Journal of Ecology, 67(2): 611-628.

Okin G., Gillette, D., Herrick, J. 2006. Multi-scale controls on and consequences of aeolian processes in landscape change in aridand semi-arid environments. Journal of Arid Environments, 65(2): 253-275.

Oliver, J. 1960. Wind and vegetation in the Dale Peninsula. Field Studies Journal, 1(2): 37-48.

Peláez J., Peñaranda, M., Herrera, H., Sierra, L., Gil, L., Mesa, J., Rodríguez, L., Sandoval, A., Grecco, A., Aramburo, J., Zuluaga, J., Trujillo, R. 2004. Parque Eólico Jepírachi: 
planeamiento y construcción del proyecto. Revista Empresas Públicas de Medellín, 15(1):101-166.

Pinilla, A., Rodríguez, L., Trujillo, R. 2009. Performance evaluation of Jepirachi Wind Park. Renewable Energy, 34(1): 48-52.

Rangel, J.O. 2012. La vegetación de la región Caribe de Colombia: composición florística y aspectos de la estructura. En: Rangel J.O. (ed.). Colombia Diversidad Biótica XII: la región Caribe de Colombia. Instituto de Ciencias Naturales, Bogotá.

Rieger, W. 1976. Vegetations Kundliche untersuchungen auf Guajira-Halbinsel (Nordost-Kolumbien). Gießener Geographische Schriften, 40: 1-142.
Rivas-Martínez, S. 2008. Clasificación bioclimática de la tierra. Centro de Investigaciones Fitosociológicas. http://www.globalbioclimatics.org/book/bioc/global bioclimatics-2008_00.htm; fecha de consulta: julio 30 de 2013.

Rodríguez, L.F. 2004. Energía eólica: tecnología, situación actual y perspectiva. Revista Empresas Públicas de Medellín, 15(1):27-46.

Váczy, C. 1980. Lexicon Botanicum Polyglottum. Editura Științifică şi Enciclopedică,Bucuresti.

Unidad de Planeación Minero Energética (UPME), Instituto de Hidrología, Meteorología y Estudios Ambientales (IDEAM). 2006. Atlas de viento y energía eólica de Colombia. Imprenta Nacional, Bogotá. 